\title{
On some operators for $p$-adic uniformly differentiable functions
}

\author{
By Katsumi SHIRATANI
}

(Received January 20, 1976)

\section{§1. Introduction}

The purpose of this paper is to give two theorems on the multiplicative theory of $p$-adic Fourier analysis. Namely we shall treat operators $\mathfrak{M}_{x}$ with Dirichlet characters $\chi$ on the $p$-adic Banach algebra of $p$-adic uniformly differentiable functions defined on the group $U_{p}$ of principal units in the rational $p$-adic number field. Then we discuss several fundamental properties of $\mathfrak{M}_{x}$. Such operators $\mathfrak{M}_{x}$ for the analytic functions on $U_{p}$ are already defined and investigated by Kubota-Leopoldt in order to introduce their $p$-adic $L$-functions [1].

Recently C. F. Woodcock [4], [5] gave an additive theory of $p$-adic Fourier analysis for $p$-adic Lipschitz functions. But we need the multiplicative theory to explain both the classical congruences of Kummer for the Bernoulli numbers and the modules of continuity of the $p$-adic $L$-functions clearly, and hence to extend our previous results [3] about the Bernoulli numbers to the ones for more general $p$-adic functions.

\section{§2. Preliminaries}

Let $Q_{p}$ be the rational $p$-adic number field and $Z_{p}$ the ring of all rational $p$-adic integers. The ring of rational integers is denoted by $Z$. We set $q=p$ for the prime $p>2$ and $q=4$ for $p=2$, and we use the normalized exponential valuation $\nu_{p}$ of $Q_{p}$ such that $\nu_{p}(p)=1$ and extend it to a valuation on the completion $\Omega_{p}$ of the algebraic closure of $Q_{p}$. The ring of integers in $\Omega_{p}$ is written by $O_{a_{p}}$.

We define a norm for $\Omega_{p}$-valued continuous functions $f$ defined on $Z_{p}$ by

$$
\nu(f)=\min _{x \in z_{p}} \nu_{p}(f(x)) .
$$

Then we know that all such functions form a $p$-adic Banach algebra $\mathscr{C}\left(Z_{p}, \Omega_{p}\right)$ under the pointwise operations and the above norm. 
Now, we set

$$
U_{p}=\left\{u \in Q_{p}^{*} ; \nu_{p}(u-1) \geqq \nu_{p}(q)\right\} .
$$

Then $U_{p}$ is the group of all principal units of $Q_{p}(p>2)$ or its subgroup of index $2(p=2)$.

The correspondence

$$
u \in U_{p} \longleftrightarrow x=\frac{\log u}{\log (1+q)} \in Z_{p}
$$

gives an isomorphism of the multiplicative group $U_{p}$ and the additive group $Z_{p}$ algebraically and topologically. Hence there is an isomorphism between the $p$-adic Banach algebra $\mathscr{C}\left(U_{p}, \Omega_{p}\right)$ under the norm $\nu(f)=\min _{u \in U_{p}} \nu_{p}(f(u))$ for $f \in \mathscr{C}\left(U_{p}, \Omega_{p}\right)$ and the $p$-adic Banach algebra $\mathscr{C}\left(Z_{p}, \Omega_{p}\right)$.

By a Lipschitz function $f \in \mathscr{C}\left(U_{p}, \Omega_{p}\right)$ we mean a function for which there exists a real constant $R$ such that $\nu_{p}\left(\frac{f(u)-f(v)}{u-v}\right) \geqq R$ holds for all $u$, $v \in U_{p}, u \neq v$. If we set

$$
R(f)=\inf _{\substack{u, v \in U_{p} \\ u \neq v}} \nu_{p}\left(\frac{f(u)-f(v)}{u-v}\right)
$$

and define a norm $V(f)$ for a Lipschitz function $f$ by

$$
V(f)=\min (\nu(f), R(f)),
$$

then all $\Omega_{p}$-valued Lipschitz functions defined on $U_{p}$ under the pointwise operations and the norm $V$ form a $p$-adic Banach algebra $\mathscr{L}_{i p}\left(U_{p}, \Omega_{p}\right)$.

If, for a given function $f: U_{p} \rightarrow \Omega_{p}$, there exists a continuous function $\phi_{f}(u, v): U_{p} \times U_{p} \rightarrow \Omega_{p}$ such that we have for all $u, v \in U_{p}, u \neq v$

$$
\phi_{f}(u, v)=\frac{f(u)-f(v)}{u-v},
$$

then we call $f$ a uniformly differentiable function.

In this case we have

$$
R(f)=\inf _{\substack{u, v \in U p \\ u \neq v}} \nu_{p}\left(\phi_{f}(u, v)\right)=\nu\left(\phi_{f}\right)
$$

and all uniformly differentiable functions make a subalgebra of the algebra $\mathscr{L}_{i p}\left(U_{p}, \Omega_{p}\right)$. It is denoted by $\mathscr{U} \mathscr{D}\left(U_{p}, \Omega_{p}\right)$.

Now, the dual group of $U_{p}$ in the sense of Pontrjagin is isomorphic to the $p$-adic torus $T_{p}=\underset{n}{\lim } Z / p^{n} Z$ and if we take the functions $\phi_{\chi}(u)=\chi(u)$ on 
$U_{p}$ corresponding to the elements $\chi$ of $T_{p}$, then we obtain, from the additive theory of Woodcock and by the isomorphism (3), a Fourier series expansion of $f \in \mathscr{U} \mathscr{D}\left(U_{p}, \Omega_{p}\right)$

$$
f(u)=\sum_{x} M_{0}\left(f \phi_{\bar{z}}\right) \phi_{x}(u) .
$$

Herein we denote by $M_{0}\left(f \phi_{\bar{z}}\right)$, simply written by $M_{\bar{z}}(f)$, the integral

$$
\begin{aligned}
M_{0}\left(f \phi_{\bar{z}}\right) & =I_{0}\left(f\left((1+q)^{x}\right) \phi_{\bar{z}}\left((1+q)^{x}\right)\right) \\
& =\lim _{\rho \rightarrow \infty} \frac{1}{p^{\rho}} \sum_{i=0}^{p \rho-1} f\left((1+q)^{i}\right) \phi_{\bar{z}}\left((1+q)^{i}\right),
\end{aligned}
$$

and the sum over $\chi$ means the limit of partial sums over all elements $\chi$ in the cyclic subgroup $T_{p}^{(n)}$ of order $p^{n}$ of $T_{p}$ under letting $n$ tend to the infinity. Finally $\bar{\chi}$ denotes the inverse character $\chi^{-1}$ of $\chi$.

In the above we note that $\chi$ can be regarded as a Dirichlet character of the second kind, namely such character of the conductor $p$-power, that satisfies $\chi(x)=\chi(\langle x\rangle)$ for the canonical decomposition $x=\omega(x)\langle x\rangle$ of $x \in Z$, $(x, p)=1$ with $\langle x\rangle \in U_{p}$, where $\omega(x)=\lim _{\rho \rightarrow \infty} x^{p \rho}$ for $p>2$ or $\omega(x)= \pm 1 \equiv x(\bmod 4)$ for $p=2$. We call any other Dirichlet character to be not of the second kind, namely it does not satisfy these conditions. Furthermore we see at once $\phi_{x}(u) \in \mathscr{U} \mathscr{D}\left(U_{p}, \Omega_{p}\right)$ and $\phi_{x}^{\prime}(u)=0$.

\section{§3. The operator $\mathfrak{M}_{x}$}

Let $\chi$ be a primitive Dirichlet character with conductor $\mathfrak{f}_{x}=\mathfrak{f}$.

For any $f \in \mathscr{U} \mathscr{D}\left(U_{p}, \Omega_{p}\right)$ and any non-negative rational integer $\rho$ we set

$$
\mathfrak{M}_{x}^{(\rho)}(f)=\frac{1}{\mathfrak{f} p^{\rho}} \sum_{x=1}^{\mathfrak{f} p} * \chi(x) f(\langle x\rangle),
$$

where $*$ denotes to take sum over all integers prime to $p$ in the given range.

First we give an elementary lemma as follows.

Lemma. If $f \in \mathscr{C}\left(U_{p}, \Omega_{p}\right)$, then we have

$$
\lim _{\rho \rightarrow \infty} \sum_{i=1}^{\mathrm{f} \rho} * \chi(i) f(\langle i\rangle)=0 \text {. }
$$

Proof. Take a $p$-power $p^{\mu}$ arbitrarily and fix it. By continuity of $f$ there is a bound $\sigma_{0}$ such that $\langle i\rangle \equiv\left\langle i_{0}\right\rangle\left(\bmod p^{\sigma}\right)$ with $\sigma \geqq \sigma_{0}$ yields $f(\langle i\rangle)$ $\equiv f\left(\left\langle i_{0}\right\rangle\right)\left(\bmod p^{\mu}\right)$. Hence, for $\rho$ sufficiently large we see

$$
\sum_{i=1}^{\mathrm{f} p \rho} *(i) f(\langle i\rangle) \equiv \sum_{i_{0}=1}^{\mathrm{f} p} *\left(i_{0}\right) f\left(\left\langle i_{0}\right\rangle\right) N_{\sigma}\left(\bmod p^{\mu}\right)
$$




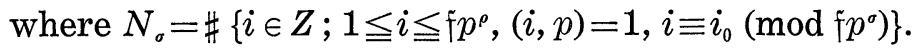

Thus we see

$$
\sum_{i=1}^{\mathfrak{f} p} *(i) f(\langle i\rangle) \equiv \sum_{i_{0}=1}^{\mathrm{f} p^{\sigma}} *\left(i_{0}\right) f\left(\left\langle i_{0}\right\rangle\right) p^{\rho-\sigma}\left(\bmod p^{\mu}\right),
$$

from which we have for $\rho$ sufficiently large

$$
\sum_{i=1}^{\mathrm{f} p} * \chi(i) f(\langle i\rangle) \equiv 0\left(\bmod p^{\mu}\right) .
$$

This means our assertion.

Now, from the definition we see readily

$$
\begin{aligned}
\mathfrak{M}_{x}^{(\rho+1)}(f)= & \frac{1}{\mathfrak{f} p^{\rho+1}} \sum_{x=1}^{\mathfrak{f} \rho+1} \chi(x) f(\langle x\rangle) \\
= & \frac{1}{\mathfrak{f} p^{\rho+1}} \sum_{x_{\rho}=1}^{\mathfrak{f} p \rho} \sum_{y=0}^{p-1} \chi\left(x_{\rho}\right)\left\{f\left(\left\langle x_{\rho}\right\rangle+\bar{\omega}\left(x_{\rho}\right) \mathfrak{f} p^{\rho} y\right)-f\left(\left\langle x_{\rho}\right\rangle\right)\right\} \\
& +\frac{1}{\mathfrak{f} p^{\rho}} \sum_{x_{\rho}=1}^{\mathfrak{f} p \rho} \chi\left(x_{\rho}\right) f\left(\left\langle x_{\rho}\right\rangle\right),
\end{aligned}
$$

where $\bar{\omega}$ means also the inverse character $\omega^{-1}$ of $\omega$.

Therefore we have

$$
\begin{aligned}
& \mathfrak{M}_{x}^{(\rho+1)}(f)-\mathfrak{M}_{x}^{(\rho)}(f)
\end{aligned}
$$

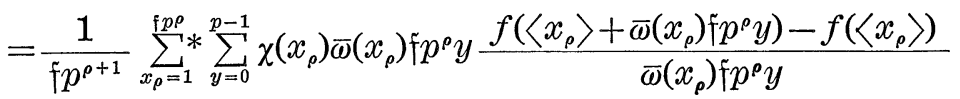

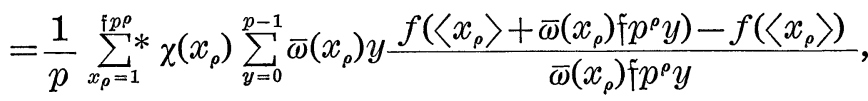

and we see

$$
\begin{aligned}
\mathfrak{M}_{x}^{(\rho+1)}(f)-\mathfrak{M}_{\alpha}^{(\rho)}(f) & \\
= & \frac{1}{p} \sum_{x_{\rho}=1}^{f p^{\rho}} *\left(x_{\rho}\right) \sum_{y=0}^{p-1} \bar{\omega}\left(x_{\rho}\right) y \phi_{f}\left(\left\langle x_{\rho}\right\rangle+\bar{\omega}\left(x_{\rho}\right) \tilde{\uparrow} p^{\rho} y,\left\langle x_{\rho}\right\rangle\right) \\
= & \frac{1}{p} \sum_{x_{\rho}=1}^{f p \rho} *\left(x_{\rho}\right) \sum_{y=0}^{p-1} \bar{\omega}\left(x_{\rho}\right) y \phi_{f}\left(\left\langle x_{\rho}\right\rangle,\left\langle x_{\rho}\right\rangle\right) \\
& +\frac{1}{p} \sum_{x_{\rho}=1}^{f p \rho} *\left(x_{\rho}\right) \sum_{y=0}^{p-1} \bar{\omega}\left(x_{\rho}\right) y\left\{\phi_{f}\left(\left\langle x_{\rho}\right\rangle+\bar{\omega}\left(x_{\rho}\right)\left\{p^{\rho} y,\left\langle x_{\rho}\right\rangle\right)-\phi_{f}\left(\left\langle x_{\rho}\right\rangle,\left\langle x_{\rho}\right\rangle\right)\right\} .\right.
\end{aligned}
$$

Because we have $\phi_{f}(u, u), \phi_{f}\left(u+\bar{\omega}(x) \uparrow p^{\rho} y, u\right) \in \mathscr{C}\left(U_{p}, \Omega_{p}\right)$ we conclude by Lemma and uniform continuity of $\phi_{f}(u, v)$ that $\mathfrak{M}_{x}^{(\rho+1)}(f)-\mathfrak{M}_{x}^{(\rho)}(f) \rightarrow 0$ as $\rho \rightarrow \infty$.

Furthermore we have 


$$
\nu_{p}\left(\mathfrak{M}_{x}^{(\rho+1)}(f)-\mathfrak{M}_{x}^{(\rho)}(f)\right) \geqq R(f)-1
$$

for any $\rho$. It follows from this that the operator $\mathfrak{M}_{x}$ defined by

$$
\mathfrak{M}_{x}(f)=\lim _{\rho \rightarrow \infty} \mathfrak{M}_{x}^{(\rho)}(f)
$$

is a bounded operator, and hence a continuous operator on $\mathscr{U} \mathscr{D}\left(U_{p}, \Omega_{p}\right)$.

\section{§4. Fundamental properties of $\mathfrak{M}_{x}$}

For $c \in Z,(c, \mathfrak{f})=1$ and $f \in \mathscr{U} \mathscr{D}\left(U_{p}, \Omega_{p}\right)$ we define $f^{c}(u)=f(\langle c\rangle u)$. Then we see $f^{c} \in \mathscr{U} \mathscr{D}\left(U_{p}, \Omega_{p}\right)$. By the condition $c x=x_{\rho}+\left\lceil p^{\rho} r_{\rho}\left(x_{\rho}\right)\right.$ with $1 \leqq x_{\rho} \leqq\left\lceil p^{\rho}\right.$, $1 \leqq x \leqq\left\lceil p^{\rho}\right.$ the numbers $r_{\rho}\left(x_{\rho}\right) \in Z$ depending on $c$ are well determined. We have easily

$$
\begin{aligned}
& \mathfrak{M}_{x}^{(\rho)}\left(f^{c}\right)=\bar{\chi}(c) \frac{1}{\mathfrak{f} p^{\rho}} \sum_{x_{\rho}=1}^{\mathfrak{f} p \rho} *\left(x_{\rho}\right) f\left(\left\langle x_{\rho}\right\rangle+\bar{\omega}\left(x_{\rho}\right) \mathfrak{f} p^{\rho} r_{\rho}\left(x_{\rho}\right)\right) \\
& =\bar{\chi}(c) \mathfrak{M}_{\alpha}^{(\rho)}(f) \\
& +\bar{\chi}(c) \frac{1}{\mathfrak{f} p^{\rho}} \sum_{x_{\rho}=1}^{\mathfrak{f} p \rho} \chi\left(x_{\rho}\right) \mathfrak{\uparrow} p^{\rho} \bar{\omega}\left(x_{\rho}\right) r_{\rho}\left(x_{\rho}\right) \phi_{f}\left(\left\langle x_{\rho}\right\rangle+\bar{\omega}\left(x_{\rho}\right) \mathfrak{f} p^{\rho} r_{\rho}\left(x_{\rho}\right),\left\langle x_{\rho}\right\rangle\right) .
\end{aligned}
$$

Therefore we obtain from Lemma as before

$$
\mathfrak{M}_{\chi}\left(f^{c}\right)=\bar{\chi}(c) \mathfrak{M}_{\mathrm{x}}(f)+\bar{\chi}(c) \lim _{\rho \rightarrow \infty} \sum_{x_{\rho}=1}^{\mathfrak{f} p \rho} \chi\left(x_{\rho}\right) \bar{\omega}\left(x_{\rho}\right) r_{\rho}\left(x_{\rho}\right) f^{\prime}\left(\left\langle x_{\rho}\right\rangle\right) .
$$

In the case $p=2$ we readily see

$$
\begin{aligned}
\lim _{\rho \rightarrow \infty} & \sum_{x_{\rho}=1}^{\mathrm{f}^{\rho}} *\left(x_{\rho}\right) \bar{\omega}\left(x_{\rho}\right) r_{\rho}\left(x_{\rho}\right) f^{\prime}\left(\left\langle x_{\rho}\right\rangle\right) \\
& =(1+\chi(-1)) \lim _{\rho \rightarrow \infty} \sum_{x_{\rho}=1}^{f 2^{\rho-1}} *\left(x_{\rho}\right) \bar{\omega}\left(x_{\rho}\right) r_{\rho}\left(x_{\rho}\right) f^{\prime}\left(\left\langle x_{\rho}\right\rangle\right) .
\end{aligned}
$$

Consequently we obtain to $f \in \mathscr{U} \mathscr{D}\left(U_{p}, \Omega_{p}\right)$ such that $f^{\prime}(u) \in O_{\Omega_{p}}$ for any $u \in U_{p}$

$$
\chi(c) \mathfrak{M}_{x}\left(f^{c}\right) \equiv \mathfrak{M}_{x}(f)\left(\bmod p^{-1} q\right) .
$$

If $\mathrm{f}$ is a $p$-power, then $\chi$ can be extended to a function on $Z_{p}$ naturally and $\chi(c)$ for $c \in Z_{p},(c, \mathfrak{f})=1$ is well determined. By the same argument as above, but using $c x=x_{\rho}+{ }^{f} p^{\rho} r_{\rho}\left(x_{\rho}\right)$ with $x, x_{\rho} \in Z, r_{\rho}\left(x_{\rho}\right) \in Z_{p}$, we conclude the congruence (18) for any such $c$. Thus, take $c=\zeta_{p-1}$ a primitive $(p-1)$-th root of unity for $p>2$ and notice $\mathfrak{M}_{x}\left(f^{c}\right)=\mathfrak{M}_{x}(f)$, then we have $\left(1-\chi\left(\zeta_{p-1}\right)\right)$ $\mathfrak{M}_{x}(f) \equiv 0\left(\bmod p^{0}\right)$.

If $\chi$ is not of the second kind, then we have $\chi\left(\zeta_{p-1}\right) \neq 1$ and $1-\chi\left(\zeta_{p-1}\right)$ is 
a unit and we see $\mathfrak{M}_{\chi}(f) \equiv 0\left(\bmod p^{0}\right)$. In the case $p=2$, if $\chi$ is not of the second kind, then $\chi(-1)=-1$ and $\mathfrak{M}_{\chi}(f)=0$ hold. In either case we have $\mathfrak{M}_{\chi}(f) \equiv 0\left(\bmod p^{-1} q\right)$ for $\chi$ not of the second kind.

Next, if $\mathfrak{f}=\mathfrak{f}_{0} p^{\mu},\left(\mathfrak{f}_{0}, p\right)=1, \mathfrak{f}_{0}>1$, then the canonical decomposition $\chi$ $=\chi_{0} \chi_{1}$ holds with $\mathfrak{f}_{x_{0}}=\mathfrak{f}_{0}, \mathfrak{f}_{\chi_{1}}=p^{\mu}$.

When we set $x=x_{0} \mathfrak{f}_{0}+x_{1} p^{\mu+\rho}+\mathfrak{f} p^{\rho} r_{\rho}\left(x_{0}, x_{1}\right), 1 \leqq x_{0} \leqq p^{\mu+\rho}, 0 \leqq x_{1} \leqq \mathfrak{f}_{0}-1$, $1 \leqq x \leqq \mathfrak{f} p^{\rho}$, the numbers $r_{\rho}\left(x_{0}, x_{1}\right) \in Z$ are also well determined. The condition $(x, p)=1$ is equivalent to $\left(x_{0}, p\right)=1$. Then we have similarly as above

$$
\langle x\rangle=\left\langle x_{0} \tilde{\digamma}_{0}\right\rangle+\bar{\omega}\left(x_{0} \mathfrak{f}_{0}\right)\left(x_{1} p^{\mu+\rho}+\mathfrak{f} p^{\rho} r_{\rho}\left(x_{0}, x_{1}\right)\right),
$$

and

$$
\begin{aligned}
\mathfrak{M}_{x}^{(\rho)}(f)= & \frac{1}{\mathfrak{f} p^{\rho}} \sum_{x_{0}=1}^{p^{\mu+\rho}} \sum_{x_{1}=0}^{\mathfrak{f}_{0}-1} \chi_{0}\left(x_{1} p^{\mu+\rho}\right) \chi_{1}\left(x_{0} \mathfrak{f}_{0}\right) f\left(\left\langle x_{0} \mathfrak{f}_{0}\right\rangle+\bar{\omega}\left(x_{0} \mathfrak{f}_{0}\right)\left(x_{1} p^{\mu+\rho}+\mathfrak{f} p^{\rho} r_{\rho}\left(x_{0}, x_{0}\right)\right)\right) \\
= & \frac{1}{\mathfrak{f} p^{\rho}} \sum_{x_{1}=0}^{\mathfrak{f}_{0}-1} \chi_{0}\left(x_{1} p^{\mu+\rho}\right) \sum_{x_{0}=1}^{p^{\mu+\rho}} * \chi_{1}\left(x_{0} \mathfrak{f}_{0}\right) f\left(\left\langle x_{0} \mathfrak{f}_{0}\right\rangle+p^{\mu+\rho} \bar{\omega}\left(x_{0} \mathfrak{f}_{0}\right)\left(x_{1}+\mathfrak{f}_{0} r_{\rho}\left(x_{0}, x_{1}\right)\right)\right) \\
= & \frac{1}{\mathfrak{f} p^{\rho}} \sum_{x_{1}=0}^{\mathfrak{f}_{0}-1} \chi_{0}\left(x_{1} p^{\mu+\rho}\right) \sum_{x_{0}=1}^{p^{\mu+\rho}} \chi_{1}\left(x_{0} \mathfrak{f}_{0}\right) f\left(\left\langle x_{0} \mathfrak{f}_{0}\right\rangle\right) \\
& +\frac{1}{\mathfrak{f}_{0}} \sum_{x_{1}=0}^{\mathfrak{f}_{0}-1} \chi_{0}\left(x_{1} p^{\mu+\rho}\right) \sum_{x_{0}=0}^{p^{\mu+\rho} *} \chi_{1}\left(x_{0} \mathfrak{f}_{0}\right) \bar{\omega}\left(x_{0} \mathfrak{f}_{0}\right)\left(x_{1}+\mathfrak{f}_{0} r_{\rho}\left(x_{0}, x_{1}\right)\right) \\
& \quad \times \phi_{f}\left(\left\langle x_{0} \mathfrak{f}_{0}\right\rangle+p^{\mu+\rho} \bar{\omega}\left(x_{0} \mathfrak{f}_{0}\right)\left(x_{1}+\mathfrak{f}_{0} r_{\rho}\left(x_{0}, x_{1}\right)\right),\left\langle x_{0} \mathfrak{f}_{0}\right\rangle\right), \\
= & \frac{1}{\mathfrak{f}_{0}} \sum_{x_{0}=1}^{p^{\mu+\rho}} \sum_{x_{1}=0}^{\mathfrak{f}_{0}-1} \chi_{0}\left(x_{1} p^{\mu+\rho}\right) \chi_{1}\left(x_{0} \mathfrak{f}_{0}\right) \bar{\omega}\left(x_{0} \mathfrak{f}_{0}\right)\left(x_{1}+\mathfrak{f}_{0} r_{\rho}\left(x_{0}, x_{1}\right)\right) \\
& \times \phi_{f}\left(\left\langle x_{0} \mathfrak{f}_{0}\right\rangle+p^{\mu+\rho} \bar{\omega}\left(x_{0} \mathfrak{f}_{0}\right)\left(x_{1}+\mathfrak{f}_{0} r_{\rho}\left(x_{0}, x_{1}\right)\right),\left\langle x_{0} \mathfrak{f}_{0}\right\rangle\right) .
\end{aligned}
$$

Hence we have

$$
\mathfrak{M}_{\chi}(f)=\lim _{\rho \rightarrow \infty} \sum_{x_{1}=0}^{\mathfrak{f}_{0}-1} \chi_{0}\left(x_{1} p^{\mu+\rho}\right) \sum_{x_{0}=1}^{p^{\mu+\rho}} * \chi_{1}\left(x_{0} \tilde{f}_{0}\right) r_{\rho}\left(x_{0}, x_{1}\right) f^{\prime}\left(\left\langle x_{0} \mathfrak{f}_{0}\right\rangle\right)
$$

In the case $p=2$ we see moreover

$$
\mathfrak{M}_{\chi}(f)=\lim _{\rho \rightarrow \infty}(1+\chi(-1)) \sum_{x_{1}=0}^{\mathfrak{f}_{0}-1} \chi_{0}\left(x_{1} p^{\mu+\rho}\right) \sum_{x_{0}=1}^{2 \mu+\rho-1} \chi_{1}\left(x_{0} \mathfrak{f}_{0}\right) \bar{\omega}\left(x_{0} \mathfrak{f}_{0}\right) r_{\rho}\left(x_{0}, x_{1}\right) f^{\prime}\left(\left\langle x_{0} \mathfrak{f}_{0}\right\rangle\right) .
$$

Consequently, if $f^{\prime}(u) \in O_{\Omega_{p}}$ for any $u \in U_{p}$, then we obtain

$$
\mathfrak{M}_{\chi}(f) \equiv 0\left(\bmod p^{-1} q\right) \text {. }
$$

For a character $\chi$ of the second kind and $p>2$ we set $x=\zeta_{p-1}^{\alpha}(1+p)^{\beta}$ $+p^{\rho} R_{\rho}(\alpha, \beta), 0 \leqq \alpha \leqq p-2,0 \leqq \beta \leqq p^{\rho-1}-1,1 \leqq x \leqq p^{\rho}$ and determine $R_{\rho}(\alpha, \beta) \in Z_{p}$. 
Then we have

$$
\begin{aligned}
& \mathfrak{M}_{\chi}^{(\rho)}(f)=\frac{p-1}{p^{\rho}} \sum_{\beta=0}^{p \rho-1-1} \chi\left((1+p)^{\beta}\right) f\left((1+p)^{\beta}\right) \\
& \quad+\sum_{\alpha=0}^{p-2} \sum_{\beta=0}^{p \rho-1-1} \zeta_{p-1}^{-\alpha} \chi\left((1+p)^{\beta}\right) R_{\rho}(\alpha, \beta) \phi_{f}\left((1+p)^{\beta}+p^{\rho} \zeta_{p-1}^{-\alpha} R_{\rho}(\alpha, \beta),(1+p)^{\beta}\right) .
\end{aligned}
$$

Hence we have also

$$
\begin{aligned}
\mathfrak{M}_{\chi}(f)= & \frac{p-1}{p} I_{0}\left(\chi\left((1+p)^{x}\right) f\left((1+p)^{x}\right)\right) \\
& +\lim _{\rho \rightarrow \infty} \sum_{\alpha=0}^{p-2} \sum_{\beta=0}^{p^{\rho-1}-1} \zeta_{p-1}^{-\alpha} \chi\left((1+p)^{\beta}\right) R_{\rho}(\alpha, \beta) f^{\prime}\left((1+p)^{\beta}\right) .
\end{aligned}
$$

Thus, for $f \in \mathscr{U} \mathscr{D}\left(U_{p}, \Omega_{p}\right)$ such that $f^{\prime}(u) \in O_{\Omega_{p}}$ for any $u \in U_{p}$ we obtain

$$
\mathfrak{M}_{\chi}(f) \equiv \frac{p-1}{p} M_{\chi}(f)\left(\bmod p^{0}\right)
$$

Similarly in the case $p=2$ we have

$$
\begin{aligned}
\mathfrak{M}_{\chi}(f)= & \frac{1}{2} I_{0}\left(\chi\left(5^{x}\right) f\left(5^{x}\right)\right) \\
& +\lim _{\rho \rightarrow \infty}(1+\chi(-1)) \sum_{\beta=0}^{2 \rho-2-1} \chi\left(5^{\beta}\right) R_{\rho}(0, \beta) f^{\prime}\left(5^{\beta}\right) .
\end{aligned}
$$

Namely, we have for $f^{\prime}(u) \in O_{s_{p}}$ for any $u \in U_{p}$

$$
\mathfrak{M}_{\chi}(f) \equiv \frac{1}{2} M_{\chi}(f)(\bmod 2) \text {. }
$$

In the both cases we obtain finally

$$
\mathfrak{M}_{\chi}(f) \equiv \frac{p-1}{p} M_{\chi}(f)\left(\bmod p^{-1} q\right) .
$$

We summarize our results in the following

THEOREM 1. Let $f$ be any function in $\mathscr{U} \mathscr{D}\left(U_{p}, \Omega_{p}\right)$ such that $f^{\prime}(u) \in O_{a_{p}}$ for each $u \in U_{p}$. Then we have the congruences:

$$
\mathfrak{M}_{\chi}(f) \equiv 0\left(\bmod p^{-1} q\right) \text { if } \chi \text { is not of the second kind, }
$$

$$
\mathfrak{M}_{\chi}(f) \equiv \frac{p-1}{p} M_{\chi}(f)\left(\bmod p^{-1} q\right) \text { if } \chi \text { is of the second kind. }
$$


For any $c \in Z,(c, f)=1$ it holds that

$$
\chi(c) \mathfrak{M}_{\chi}\left(f^{c}\right) \equiv \mathfrak{M}_{\chi}(f)\left(\bmod p^{-1} q\right) .
$$

Furthermore, when the function $f(u)$ is multiplicative, i.e. $f(u v)$ $=f(u) f(v)$ for any $u, v \in U_{p}$, we have the following Theorem 2.

From the definition we have

$$
\begin{aligned}
M_{\chi}\left(f^{1+p}\right) & =M_{\chi}(f)+\lim _{\rho \rightarrow \infty} \frac{1}{p^{\rho}}\left\{f\left((1+p)^{p^{\rho}}\right)-f(1)\right\} \\
& =M_{\chi}(f)+\lim _{\rho \rightarrow \infty} \frac{f\left((1+p)^{p \rho}\right)-f(1)}{(1+p)^{p^{\rho}}-1} \frac{(1+p)^{p^{\rho}}-1}{p^{\rho}} .
\end{aligned}
$$

Therefore we have

$$
M_{\chi}\left(f^{1+p}\right)=M_{\chi}(f)+f^{\prime}(1) \log (1+p) .
$$

By virtue of the multiplicative property $M_{\chi}\left(f^{1+p}\right)=\chi(1+p) f(1+p) M_{\chi}(f)$ it follows that

$$
(1-\chi(1+p) f(1+p)) M_{\chi}(f)=-f^{\prime}(1) \log (1+p) .
$$

In the case $p=2$ quite similarly it also holds :

$$
(1-\chi(1+q) f(1+q)) M_{\chi}(f)=-f^{\prime}(1) \log (1+q) .
$$

Thus we obtain

THEOREM 2. For any multiplicative function $f \in \mathscr{U} \mathscr{D}\left(U_{p}, \Omega_{p}\right)$ such that $f(u), f^{\prime}(u) \in O_{a_{p}}$ for each $u \in U_{p}$ we have

$$
(1-\chi(1+q) f(1+q)) \mathfrak{M}_{\chi}(f) \equiv 0\left(\bmod p^{-1} q\right) .
$$

This is a generalization of determination of the denominators of the Bernoulli numbers.

\section{§5. Examples}

1) As usual we define a linear difference operator $\Delta$ for any sequence $\left\{a_{m}\right\}$ in $\Omega_{p}$ by $\Delta a_{m}=a_{m+1}-a_{m}$.

We take the function $f(u)=\frac{1}{q^{k}} \Delta^{k} \frac{1}{m} u^{m}$ for $m \geqq 1$, where $k$ denotes an arbitrarily fixed non-negative rational integer. Then the formulas (30), (31) in Theorem 1 reduce simply to the known congruences of Kummer.

In fact we see 


$$
f^{\prime}(u)=\frac{1}{q^{k}} \Delta^{k} u^{m-1}=\frac{1}{q^{k}} u^{m-1}(u-1)^{k} \in Z_{p}
$$

and

$$
\mathfrak{M}_{x}(f)=\frac{1}{q^{k}} \Delta^{k} \mathfrak{M}_{x}\left(\frac{1}{m} u^{m}\right)=\frac{1}{q^{k}} \Delta^{k} \frac{1}{m} D_{x^{\omega}-m}^{m},
$$

where we put $D_{\chi \omega^{-m}}^{m}=-\left(1-\chi \omega^{-m}(p) p^{m-1}\right) B_{x^{-m}}^{m}$ with the $m$-th generalized Bernoulli number $B_{x^{\omega}-m}^{m}[3]$.

2) If we take $f(u)=-\frac{1}{1-s} u^{1-s}, s \in Z_{p}, s \neq 1$, then Theorem 1 means a determination of the exact modules of continuity of $p$-adic $L$-functions of Kubota-Leopoldt. Namely, we see

$$
f^{\prime}(u)=-\frac{1}{1-s} \frac{d}{d u}\left(e^{(1-s) \log u}\right)=-u^{1-s} u^{-1}=-u^{-s} \in Z_{p},
$$

and

$$
\mathfrak{M}_{\chi}(f)=-\frac{1}{1-s} \mathfrak{M}_{\chi}\left(u^{1-s}\right)=L_{p}(s, \chi)
$$

by the definition of the $p$-adic $L$-functions.

Thus we can know immediately:

$$
\begin{gathered}
L_{p}(s, \chi) \equiv 0\left(\bmod p^{-1} q\right) \quad \text { for } \chi \text { not of the second kind, } \\
L_{p}(s, \chi) \equiv-\frac{u_{p}}{1-\chi(1+q)(1+q)^{1-s}}\left(\bmod p^{-1} q\right)
\end{gathered}
$$

with a constant $u_{p}=-\frac{p-1}{p} \log (1+q)$ for $\chi$ of the second kind.

But these facts are already well known [2], [3].

3) When we select $f(u)$ as $f(u)=\phi_{\chi_{2}}(u)=\chi_{2}(u)$ with any Dirichlet character $\chi_{2}$ of the second kind, we have $f^{\prime}(u)=0$. Therefore all the congruences in Theorems 1, 2 are automatically equalities as can be seen in the preceding section. Thus we have

$$
\mathfrak{M}_{x}\left(\phi_{x_{2}}\right)=0 \quad \text { for } \chi \text { not of the second kind, }
$$$$
\mathfrak{M}_{\chi}\left(\phi_{x_{2}}\right)=\frac{p-1}{p} M_{\chi}\left(\phi_{x_{2}}\right) \quad \text { for } \chi \text { of the second kind, }
$$

$$
M_{\chi}\left(\phi_{x_{2}}\right)=I_{0}\left(\phi_{x x_{2}}\right)= \begin{cases}0 & \text { for } \chi \neq \bar{\chi}_{2}, \\ 1 & \text { for } \chi=\bar{\chi}_{2} .\end{cases}
$$


4) Let $\chi$ be a Dirichlet character of the second kind. Multiplying $\bar{\chi}(u)$ to the both hand sides in (25) and summing over the elements in $T_{p}^{(n)}$ we have in the case $p \neq 2$

$$
\begin{aligned}
\sum_{\chi \in T_{p}^{(n)}} \mathfrak{M}_{x}(f) \bar{\chi}(u)= & \frac{p-1}{p} \sum_{\chi \in T_{p}^{(n)}} I_{0}\left(f\left((1+p)^{x}\right) \phi_{x}\left((1+p)^{x}\right)\right) \phi_{\bar{\chi}}(u) \\
& +\lim _{\rho \rightarrow \infty} \sum_{\alpha=0}^{p-2} \zeta_{p-1}^{-\alpha} \sum_{\substack{0 \leqslant \beta p \\
(1+p) \beta=\alpha}} p^{n} R_{\rho}(\alpha, \beta) f^{\prime}\left((1+p)^{\beta}\right) .
\end{aligned}
$$

Consequently we have

$$
\sum_{x \in T_{p}} \mathfrak{M}_{\chi}(f) \phi_{\bar{z}}(u)=\frac{p-1}{p} \sum_{x \in T_{p}} I_{0}\left(f\left((1+p)^{x}\right) \phi_{x}\left((1+p)^{x}\right)\right) \phi_{\bar{z}}(u)
$$

Note here that we regard $\psi(x)=\phi_{x}\left((1+p)^{x}\right)$ as a character of the additive group $Z_{p}$. Hence the sum

$$
\sum_{x \in T_{p}} I_{0}\left(f\left((1+p)^{x}\right) \phi_{x}\left((1+p)^{x}\right)\right) \phi_{\bar{\chi}}(u)
$$

is a Fourier series expansion of the function $g(x)=f\left((1+p)^{x}\right)$.

By making use of Woodcock's theory [4] we obtain

$$
\sum_{x \in T_{p}} \mathfrak{M}_{x}(f) \phi_{\bar{x}}(u)=\frac{p-1}{p} f(u) .
$$

In the case $p=2$ we have quite the same.

In particular, if we take $f(u)=-\frac{1}{1-s} u^{1-s}$, then we conclude from the above

$$
-\frac{1}{1-s} u^{1-s}=\frac{p}{p-1} \sum_{x \in T_{p}} L_{p}(s, \chi) \phi_{\bar{\chi}}(u)
$$

\section{References}

[1] T. Kubota and H. W. Leopoldt, Eine $p$-adische Theorie der Zetawerte: I, J. Reine Angew. Math., 214/215 (1964), 328-339.

[2] J.-P. Serre, Formes modulaires et fonctions zêta $p$-adiques, Lecture Notes in Math., 350 (1973), 191-268.

[3] K. Shiratani, Kummer's congruence for generalized Bernoulli numbers and its application, Mem. Fac. Sci. Kyushu Univ. Ser. A, 26 (1972), 119-138.

[4] C. F. Woodcock, Fourier analysis for p-adic Lipschitz functions, J. London Math. Soc., 7 (1974), 681-693. 
[5] C. F. Woodcock, An invariant $p$-adic integral on $Z_{p}$, J. London Math. Soc., 8 (1974), 731-734.

Department of Mathematics

KYUSHU UNIVERSITY

812 FUKUOKA 